\title{
The nature of molecular cloud boundary layers from SOFIA [O I] observations
}

\author{
W. D. Langer ${ }^{1}$, P. F. Goldsmith ${ }^{1}$, J. L. Pineda ${ }^{1}$, E. T. Chambers ${ }^{2}$, K. Jacobs ${ }^{3}$, and H. Richter ${ }^{4}$ \\ ${ }^{1}$ Jet Propulsion Laboratory, California Institute of Technology, 4800 Oak Grove Drive, Pasadena, CA 91109, USA \\ e-mail: William.Langer@jpl.nasa.gov \\ 2 SOFIA-USRA, NASA Ames Research Center, MS 232-12, Moffett Field, CA 94035-0001, USA \\ ${ }^{3}$ I. Physikalisches Institut der Universität zu Köln, Zülpicher Strasse 77, 50937 Köln, Germany \\ ${ }^{4}$ DLR, Institut für Optische Sensorsysteme, Rutherfordstraße 2, 12489 Berlin-Adlershof, Germany
}

Received 23 January 2018 / Accepted 13 July 2018

\begin{abstract}
Context. Dense highly ionized boundary layers (IBLs) outside of the neutral Photon Dominated Regions (PDRs) have recently been detected via the 122 and $205 \mu \mathrm{m}$ transitions of ionized nitrogen. These layers have higher densities than in the Warm Ionized Medium (WIM) but less than typically found in $\mathrm{H}$ II regions. Observations of [C II] emission, which is produced in both the PDR and IBL, do not fully define the characteristics of these sources. Observations of additional probes which just trace the PDRs, such as the fine structure lines of atomic oxygen, are needed derive their properties and distinguish among different models for [C II] and [N II] emissison.

Aims. We derive the properties of the PDRs adjacent to dense highly ionized boundary layers of molecular clouds.

Methods. We combine high-spectral resolution observations of the $63 \mu \mathrm{m}$ [O I] fine structure line taken with the upGREAT HFA-band instrument on SOFIA with [C II] observations to constrain the physical conditions in the PDRs. The observations consist of samples along four lines of sight (LOS) towards the inner Galaxy containing several dense molecular clouds. We interpret the conditions in the PDRs using radiative transfer models for [C II] and [O I].

Results. We have a 3.5- $\sigma$ detection of [O I] toward one source but only upper limits towards the others. We use the [O I] to [C II] ratio, or their upper limits, and the column density of $\mathrm{C}^{+}$to estimate the thermal pressure, $P_{\text {th }}$, in these PDRs. In two LOS the thermal pressure is likely in the range $2-5 \times 10^{5}$ in units of $\mathrm{K} \mathrm{cm}^{-3}$, with kinetic temperatures of order $75-100 \mathrm{~K}$ and $\mathrm{H}_{2}$ densities, $n\left(\mathrm{H}_{2}\right) \sim 2-$ $4 \times 10^{3} \mathrm{~cm}^{-3}$. For the other two sources, where the upper limits on [O I] to [C II] are larger, $P_{\text {th }} \lesssim 10^{5}\left(\mathrm{~K} \mathrm{~cm}^{-3}\right)$. We have also used PDR models that predict the $[\mathrm{O}$ I] to [C II] ratio, along with our observations of this ratio, to limit the intensity of the Far UV radiation field. Conclusions. The [C II] and [N II] emission with either weak, or without any, evidence of [O I] indicates that the source of dense highly ionized gas traced by [N II] most likely arises from the ionized boundary layers of clouds rather than from $\mathrm{H}$ II regions.
\end{abstract}

Key words. ISM: clouds - ISM: atoms - ISM: structure

\section{Introduction}

In an interstellar cloud the spatial transition from atomic to molecular gas takes place in photon dominated regions (PDRs; see reviews by Hollenbach \& Tielens 1997, 1999), which are also a source of a significant fraction of the far-infrared emission from the Milky Way and other galaxies. Exterior to the PDRs the gas makes the transition from neutral to ionized hydrogen. The ionized gas can take the form of a low density ionized boundary layer (IBL) in the case of weak UV fields, or a dense H II region in the proximity to a strong UV field arising from massive star formation. PDRs and H II regions are the boundary regions where the effects of star formation on molecular clouds manifest themselves. They have been the focus of a considerable modeling effort (see Tielens \& Hollenbach 1985; Sternberg \& Dalgarno 1989; Kaufman et al. 1999; Abel et al. 2005; Le Petit et al. 2006; Bron et al. 2018, and references therein). The observational analysis of PDRs, H II regions, and IBLs has improved considerably since the availability of far-infrared spectroscopic data from the Herschel Space Observatory (see Ossenkopf et al. 2013; Köhler et al. 2014; Stock et al. 2015; Joblin et al. 2018; Wu et al. 2018, and references therein) and the Stratospheric Observatory for Infrared Astronomy (SOFIA; e.g., Schneider et al. 2012; Pérez-Beaupuits et al. 2015; Pabst et al. 2017; Mookerjea et al. 2018) . Most of these studies of the ionized and PDR layers have focused on very bright H II regions where high UV flux, density, and temperature produce strong far-infrared emission, making such regions easily observable in key gas tracers such as the fine-structure lines of $\mathrm{C}^{+}, \mathrm{N}^{+}$, and $\mathrm{O}$. Less is known about the IBL-PDR conditions for typical molecular clouds where the UV field is smaller and, thus, the lines are weaker. The Herschel Space Observatory HIFI GOT C+ survey (Langer et al. 2010; Pineda et al. 2013) took a step in studying molecular cloud PDRs and IBLs in that it sampled [C II] along several hundred lines of sight (LOS) in the Galaxy producing an unbiased database of a few thousand clouds of various evolutionary stages with most LOS not containing H II regions as indicated by weak [C II] emission. However, because [C II] samples both weakly and highly ionized regions, there remains some uncertainty about the relative contributions of the ionized and PDR regions. Furthermore, because [C II] has only one fine-structure transition one cannot solve uniquely for the properties of the gas. For the GOT C+ survey Langer et al. (2014) derived the column density of material traced by [C II] by assuming a thermal pressure and its Galactic gradient.

In contrast to $\mathrm{C}^{+}$, ionized nitrogen, $\mathrm{N}^{+}$, arises only from highly ionized gas as its ionization potential, $14.5 \mathrm{eV}$, requires 
the UV photons with $\lambda \leq 912 \AA$, such as found in H II regions, or high temperatures, $\gtrsim 6000 \mathrm{~K}$, where electron collisional ionization or proton charge transfer maintains a high fractional ionization (Langer et al. 2015), such as in the warm ionized medium (WIM). The fine-structure lines of $\mathrm{N}^{+},[\mathrm{N}$ II $]$, at 122 and $205 \mu \mathrm{m}$, provide a probe of the highly ionized gas and having two transitions makes it possible to derive the electron/proton density using radiative transfer models because the solutions are not temperature sensitive in these environments.

To determine the density of the highly ionized gas associated with the $[\mathrm{C}$ II] detected in the GOT C+ survey, Goldsmith et al. (2015) conducted a follow up Herschel Open Time PACS [N II] survey sample along 149 LOS covering longitudes from $0^{\circ}$ to $344^{\circ}$ at a Galactic latitude of $b=0^{\circ}$, that had previously been observed in [C II] (Langer et al. 2010; Pineda et al. 2013; Langer et al. 2014). They detected 96 LOS in both transitions $(205$ and $122 \mu \mathrm{m})$ mostly at longitudes between $-60^{\circ}$ and $+60^{\circ}$. They found that the density of the highly ionized gas responsible for $[\mathrm{N} \mathrm{II}]$ is typically in the range $n(\mathrm{e}) \sim 5-50 \mathrm{~cm}^{-3}$, much higher than in the WIM $\left(\sim 0.05 \mathrm{~cm}^{-3}\right)$. To better identify the sources of highly ionized gas, the [N II] survey included ten lines of sight observed at high spectral resolution with HIFI and found over 30 distinct cloud components in nine of these (excluding the Galactic Center) as identified by their [C II] and [N II] emission (Langer et al. 2016). In addition, Goldsmith et al. (2015) and Langer et al. (2016) found that a significant fraction of the [C II], of order $20 \%-30 \%$, comes from highly ionized dense gas. A related study of the Scutum spiral arm tangency found that [N II] $205 \mu \mathrm{m}$ emission arose from two components, a low density, $n(\mathrm{e}) \sim 0.9 \mathrm{~cm}^{-3}$, compressed WIM at the arm-interarm interface and from high density, $n(\mathrm{e}) \sim 30 \mathrm{~cm}^{-3}$, ionized gas associated with the molecular clouds deep in the arm (Langer et al. 2017).

In contrast to [N II], which probes only highly ionized gas, and [C II], which probes weakly and highly ionized gas, the fine structure lines of atomic oxygen, [O I] at 63 and $146 \mu \mathrm{m}$, probe exclusively the neutral, weakly ionized PDR regions. Oxygen is readily ionized in $\mathrm{H}$ II and IBL regions as its ionization potential is only about $0.02 \mathrm{eV}$ above that of hydrogen, so that electron collisional ionization and especially, proton charge transfer are very efficient, even at low temperatures $(\gtrsim 100 \mathrm{~K})$. Therefore, in principle, by observing [O I] we can determine the properties of the neutral gas probed by [C II]. Strong [O I] emission is detected in H II regions (e.g., Boreiko \& Betz 1996; Oberst et al. 2011; Leurini et al. 2015) ([O I] is also readily detected in absorption against hot cores (Wiesemeyer et al. 2016)). However, [O I] emission will be more difficult to detect in PDRs not illuminated by strong UV sources because the ${ }^{3} \mathrm{P}_{1}$ level that gives rise to the [O I] $63-\mu \mathrm{m}$ line, lies at $227.7 \mathrm{~K}$ above the ground state (Schöier et al. 2005). In contrast, the ${ }^{3} \mathrm{P}_{3 / 2}$ upper level of $\mathrm{C}^{+}$which gives rise to the $158-\mu \mathrm{m}$ line, is only at $91.2 \mathrm{~K}$. Furthermore, using the collisional de-excitation rates at $T_{k} \sim 100 \mathrm{~K}$ (Schöier et al. 2005; Wiesenfeld \& Goldsmith 2014), the [O I] transition has a high critical density $\left(n\left(\mathrm{H}_{2}\right) \sim 6 \times 10^{5} \mathrm{~cm}^{-3}\right)$ compared to [C II] which has a much lower critical density $\left(n\left(\mathrm{H}_{2}\right) \sim 3 \times 10^{3} \mathrm{~cm}^{-3}\right)$.

To determine the nature of the neutral gas adjacent to the dense highly ionized gas seen in [N II], we proposed a SOFIA pilot program to observe the [O I] $63 \mu \mathrm{m}$ line along four LOS previously observed in [C II] and [N II] with HIFI. All sources are associated with molecular clouds as indicated by the detection of ${ }^{13} \mathrm{CO}$ (see Langer et al. 2016), and thus PDRs. We detected $[\mathrm{OI}]$ in one source at the 3.5- $\sigma$ level, but were only able to set upper limits on the [O I] emission in the remaining sources. We use the $[\mathrm{O} I]$ to $[\mathrm{C} \mathrm{II}]$ emission ratio and ratio limits to constrain the thermal pressure in the PDRs.
Table 1. Sources observed in [C II], [N II], and [O I]

\begin{tabular}{ccccc}
\hline \hline GOT C+ LOS & $l$ & $b$ & $V_{\mathrm{LSR}}{ }^{a}\left(\mathrm{~km} \mathrm{~s}^{-1}\right)$ & $\mathrm{rms}(\mathrm{K})^{b}$ \\
\hline G031.3+0.0 & 31.2766 & 0.0 & 100.0 & 0.07 \\
G049.1+0.0 & 49.1489 & 0.0 & 60.0 & 0.09 \\
G305.1+0.0 & $305^{\circ} .1060$ & 0.0 & -30.0 & 0.04 \\
G337.0+0.0 & 336.9570 & 0.0 & -75.0 & 0.03 \\
\hline
\end{tabular}

Notes. ${ }^{(a)} \mathrm{A}$ central band velocity adopted for observing purposes. ${ }^{(b)}$ For a channel width of $1.08 \mathrm{~km} \mathrm{~s}^{-1}$.

This paper is organized as follows. In Sect. 2, we present the observations, while in Sect. 3, we derive or set limits on the properties of the PDRs observed in [C II]. Section 4 summarizes the results.

\section{Observations}

We observed four lines of sight labeled G031.1+0.0, G049.1+0.0, G305.1+0.0, and G337.0+0.0, using the GOT C+ notation Gxxx.yy+x.x (Langer et al. 2014), which refers to their approximate longitude and latitude. The actual longitudes and latitudes are given in Table 1. In the case of G305.1+0.0 an atmospheric line in the $[\mathrm{O} \mathrm{I}]$ band coincided with a portion of the strongest $\left[\mathrm{C}\right.$ II] and $\left[\mathrm{N}_{\mathrm{II}}\right]$ emission from $V_{\mathrm{LSR}}=-32$ to $-18 \mathrm{~km} \mathrm{~s}^{-1}$, making it difficult to assess whether there is any [O I] emission present. Therefore, the G305.1+0.0 LOS is omitted from further detailed discussion.

We observed the neutral atomic oxygen ${ }^{3} \mathrm{P}_{1}-{ }^{3} \mathrm{P}_{2}$ fine structure line, $[\mathrm{OI}]$, at $4744.77749 \mathrm{GHz}(\lambda \sim 63 \mu \mathrm{m})$ using the upgraded German Receiver for Astronomy at Terahertz Frequencies (GREAT ${ }^{1}$; Heyminck et al. 2012) and (upGREAT ${ }^{1}$; Risacher et al. 2016) $H F A$-band 7-pixel single polarization array onboard SOFIA (Young et al. 2012). The upGREAT $H F A$ band uses NbN hot electron bolometer (HEB) waveguide mixers (Büchel et al. 2015) with a quantum cascade laser providing the local oscillator (Richter et al. 2015). The total bandwidth is $2.5 \mathrm{GHz}$ and the spectral resolution was smoothed to $1.08 \mathrm{~km} \mathrm{~s}^{-1}$ to improve signal to noise. The central $[\mathrm{O} \mathrm{I}]$ pixel was pointed towards the GOT C+ LOS.

Our program (proposal ID 05_0015; PI Langer) was part of the Guest Observer Cycle 5 campaign. The observations were made on June 14, June 28, and July 4, 2017. The data were processed with the GREAT calibrator using the atmospheric transmission model of Guan et al. (2012) to correct for atmospheric opacity. The observing times on-source for G031.3+0.0, G049.1+0.0, G305.1+0.0, and G337.0+0.0 were 4.3, 6.7, 7.3 and $7.3 \mathrm{~min}$, respectively. To get properly calibrated [O I] spectra we need to check for any emission in the reference OFF positions for every ON position at $b=0^{\circ}$. The observing limits with SOFIA allow a maximum offset of the OFF position to be $b=0.4$ (in latitude). Therefore, we used $0^{\circ} .4$ as a primary OFF position, and we observed this position using 0.8 as a secondary OFF position to check if it were free of [O I] emission. No emission was detected at the OFF positions.

The [O I] data show standing waves having a characteristic period of about $15-20 \mathrm{~km} \mathrm{~s}^{-1}$. We removed these standing waves using an asymmetric least squares smoothing approach (Eilers \& Boelens 2005) which identifies and removes a smooth baseline

\footnotetext{
1 GREAT and upGREAT are a development by the MPI für Radioastronomie and the KOSMA/Universität zu Köln in cooperation with the MPI für Sonnensystemforschung and the DLR Institut für Planetenforschung.
} 

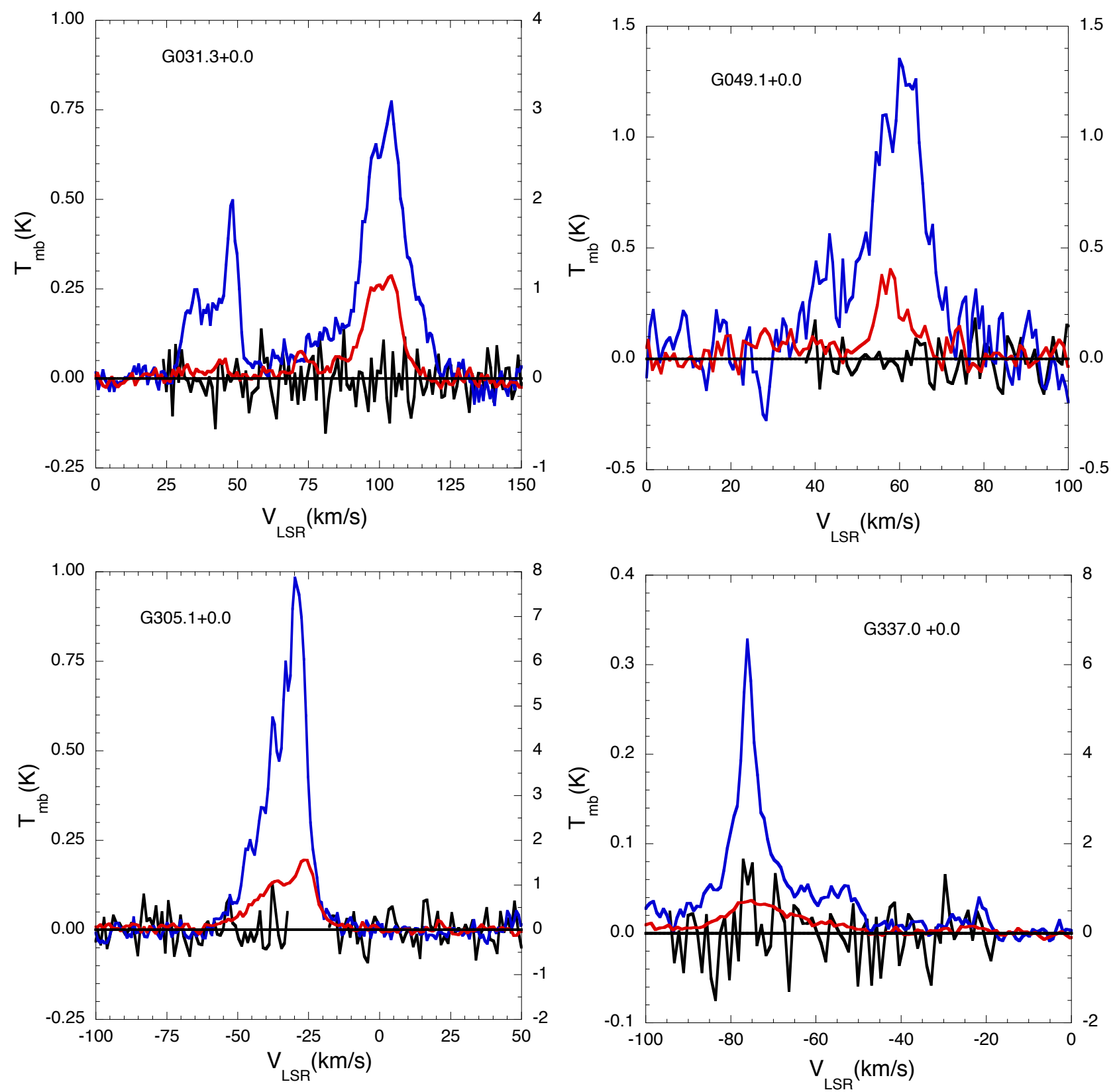

Fig. 1. Main beam temperature versus velocity for the [O I] (black) GREAT $H F A$-band spectra for four lines of sight. The scale for [O I] is on the left and the scale for [C II] and [N II] is on the right. There is a 3.5- $\sigma$ detection at G337.0+0.0, but only upper limits for the other three LOS. There is an emission gap in [O I] for G305.1+0.0 due to the presence of an atmospheric absorption line which has been removed. Superimposed on the [O I] spectra are the corresponding HIFI [C II] (blue) and [N II] (red) spectra (Langer et al. 2016).

to the data. This approach is appropriate for our data, as the [O I] line detected in G337.0+0.0 has a linewidth much smaller than the longer period standing waves.

Each pixel of the 7-pixel GREAT $H F A$-band array has a FWHM beam size of $6.3^{\prime \prime}$ at $4.7 \mathrm{THz}$. The array is arranged in a hexagonal pattern with a central beam. The beam spacing is approximately two beam widths and the array has a footprint about $27^{\prime \prime}$ across. We did not detect [O I] in any of the individual pixels, so we combined all seven [O I] pixels at each LOS to increase the sensitivity, and the resulting rms noise is listed in Table 1.

The intensities have been converted to main beam temperature, $T_{\mathrm{mb}}(\mathrm{K})$, using beam efficiencies appropriate to each pixel with a typical value of $\eta_{\mathrm{mb}}([\mathrm{OI}])=0.63$, and the data were smoothed in velocity to a channel width of $1.08 \mathrm{~km} \mathrm{~s}^{-1}$ to improve the signal-to-noise. In Fig. 1, we plot the main beam temperature, $T_{\mathrm{mb}}$ versus the LSR velocity, $V_{\mathrm{LSR}}$, for [O I] averaged over all pixels in the array, and the HIFI single pixel dual polarization [N II] and [C II] spectra (Goldsmith et al. 2015; Langer et al. 2016). The resulting averaged [O I] spectra correspond to an angular size of $\sim 27^{\prime \prime}$, somewhat larger than the HIFI [C II $], 12^{\prime \prime}$, and [N II], 15", beam sizes.

In Fig. 1, we see that there is no $[\mathrm{OI}]$ detected from $\mathrm{G} 031.3+0.0$, G049.1+0.0, and G305.1+0.0, but there is a detection at the 3.5- $\sigma$ level in G337.0+0.0 exactly at the peak of the $[\mathrm{C}$ II $]$ and $[\mathrm{N}$ II $]$ emission, $V_{\mathrm{LSR}}=-76 \mathrm{~km} \mathrm{~s}^{-1}$. In Table 1, we 
Table 2. [O I], [C II], and [N II] intensities in units of $\mathrm{K} \mathrm{km} \mathrm{s}^{-1}$.

\begin{tabular}{cccccccc}
\hline \hline GOT C+ LOS & $\begin{array}{c}V_{\mathrm{LSR}} \\
\left(\mathrm{km} \mathrm{s}^{-1}\right)\end{array}$ & $\begin{array}{c}I([\mathrm{O} \mathrm{I}]) \\
\left(\mathrm{K} \mathrm{km} \mathrm{s}^{-1}\right)\end{array}$ & $\begin{array}{c}I_{\mathrm{tot}}([\mathrm{C} \mathrm{II}]) \\
\left(\mathrm{K} \mathrm{km} \mathrm{s}^{-1}\right)\end{array}$ & $\begin{array}{c}I([\mathrm{~N} \mathrm{II}]) \\
\left(\mathrm{K} \mathrm{km} \mathrm{s}^{-1}\right)\end{array}$ & $\begin{array}{c}I_{n}([\mathrm{C} \mathrm{II}])^{a} \\
\left(\mathrm{~K} \mathrm{~km} \mathrm{~s}^{-1}\right)\end{array}$ & $I([\mathrm{O} \mathrm{I}]) / I_{n}([\mathrm{C} \mathrm{II}])$ & $I([\mathrm{~N} \mathrm{II}]) / I_{\mathrm{tot}}([\mathrm{C} \mathrm{II}])$ \\
\hline G031.3+0.0 & $25 \rightarrow 55$ & $<0.24^{b}$ & 22.4 & 2.3 & 16.3 & $<1.5 \times 10^{-2}$ & 0.10 \\
& $85 \rightarrow 120$ & $<0.29^{b}$ & 53.2 & 17.5 & 6.0 & $<4.8 \times 10^{-2}$ & 0.33 \\
G049.1+0.0 & $50 \rightarrow 75$ & $<0.31^{b}$ & 18.3 & 3.7 & 8.3 & $<3.7 \times 10^{-2}$ & 0.20 \\
$\mathrm{G} 337.0+0.0$ & $-80 \rightarrow-70$ & $0.25 \pm 0.07^{c}$ & 49.0 & 10.8 & 18.3 & $(1.4 \pm 0.4) \times 10^{-2}$ & 0.22 \\
\hline
\end{tabular}

Notes. ${ }^{(a)} I_{n}([\mathrm{C}$ II $])$ is the intensity of [C II] that arises just from the PDR. ${ }^{(b)}$ The $3-\sigma$ detection limit. ${ }^{(c)}$ The $1-\sigma$ uncertainty.

Table 3. [O I], [C II], and [N II] intensities in units of $\mathrm{W} \mathrm{m}^{-2} \mathrm{sr}^{-1}$.

\begin{tabular}{|c|c|c|c|c|c|c|c|}
\hline GOT C+ LOS & $\begin{array}{c}V_{\mathrm{LSR}} \\
\left(\mathrm{km} \mathrm{s}^{-1}\right)\end{array}$ & 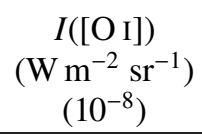 & $\begin{array}{c}I_{\text {tot }}([\mathrm{C} \mathrm{III]}) \\
\left(\mathrm{W} \mathrm{m}^{-2} \mathrm{sr}^{-1}\right) \\
\left(10^{-8}\right)\end{array}$ & $\begin{array}{c}I([\mathrm{~N} \mathrm{II}]) \\
\left(\mathrm{W} \mathrm{m}^{-2} \mathrm{sr}^{-1}\right) \\
\left(10^{-8}\right)\end{array}$ & $\begin{array}{c}I_{n}([\mathrm{C} \mathrm{III}])^{a} \\
\left(\mathrm{~W} \mathrm{~m} \mathrm{~m}^{-2} \mathrm{sr}^{-1}\right) \\
\left(10^{-8}\right)\end{array}$ & $I([\mathrm{O} \mathrm{I}]) / I_{n}([\mathrm{C} \mathrm{II}])$ & $\begin{array}{c}G_{o}{ }^{d} \\
n=2 \times 10^{3} \mathrm{~cm}^{-3}\end{array}$ \\
\hline \multirow[t]{2}{*}{$\mathrm{G} 031.3+0.0$} & $25 \rightarrow 55$ & $<2.6^{b}$ & 15.5 & 0.7 & 11.5 & $<0.23$ & $<15$ \\
\hline & $85 \rightarrow 120$ & $<3.2^{b}$ & $37.4^{b}$ & 5.6 & 4.2 & $<0.75$ & $<100$ \\
\hline G049.1+0.0 & $50 \rightarrow 75$ & $<3.4^{b}$ & 12.9 & 1.2 & 5.8 & $<0.58$ & $<55$ \\
\hline G337.0+0.0 & $-80 \rightarrow-70$ & $2.7 \pm 0.7^{c}$ & 34.5 & 3.5 & 12.9 & $(0.21 \pm 0.06)$ & 15 \\
\hline
\end{tabular}

Notes. ${ }^{(a)} I_{n}([\mathrm{C}$ II $])$ is the intensity of [C II $]$ that arises just from the PDR. ${ }^{(b)}$ The $3-\sigma$ detection limit. ${ }^{(c)}$ The $1-\sigma$ uncertainty. ${ }^{(d)} G_{o}$ is derived from the models of Kaufman et al. (1999) and is in units of the Habing field, $1.6 \times 10^{-3} \mathrm{ergs} \mathrm{cm}^{-2} \mathrm{~s}^{-1}$.

give the rms per $1.08 \mathrm{~km} \mathrm{~s}^{-1}$ channel for the [O I] observations. In Table 2, we give the integrated intensities for the far-IR lines in units appropriate for heterodyne systems, $\mathrm{K} \mathrm{km} \mathrm{s}^{-1}$, while in Table 3, we convert these to standard units of $\mathrm{W} \mathrm{m}^{-2} \mathrm{sr}^{-1}$, assuming that the source fills the beam. In Table 2, we list the 1- $\sigma$ limits on [O I] derived by integrating over the velocities corresponding to cloud components (see Langer et al. 2016) as defined by [N II] and/or [C II]. In the case of G337.0+0.0, where we detect [O I], we give the integrated intensity of the line at $-76 \mathrm{~km} \mathrm{~s}^{-1}$ and the $1-\sigma$ error.

In G337.0+0.0 the line width FWHM of [O I] $\sim 3 \mathrm{~km} \mathrm{~s}^{-1}$, as derived from a gaussian fit, slightly narrower than that of [C II], $\sim 4 \mathrm{~km} \mathrm{~s}^{-1}$, as derived from a multi-Gaussian fit (the blend of lines makes it difficult to determine $F W H M$ for the $-76 \mathrm{~km} \mathrm{~s}^{-1}$ component of $[\mathrm{N} \mathrm{II}])$.

\section{Discussion}

Atomic oxygen has two fine structure transitions, at 63 and $146 \mu \mathrm{m}$, and so, in principle, these can be used to derive two of the three fundamental parameters in the PDR consisting of density, temperature, and column density, using radiative transfer models. When combined with the [C II] emission, all three parameters can be derived if we know the carbon to oxygen ratio and if their emission arises from the same volume. However, we only have one of the [O I] emission lines, so at best we can derive constraints on the conditions in PDRs. We will use the [O I] to [C II] intensity ratio to constrain $P_{\text {th }}$ in the PDRs and, indirectly, bounds on the density and kinetic temperature.

To derive the intensity ratio $I([\mathrm{O} \mathrm{I}]) / I([\mathrm{C} \mathrm{II}])$ we start with the relationship between intensity and column density for an optically thin species (Goldsmith et al. 2012),

$I_{\mathrm{ul}}=\int T_{\mathrm{ul}} \mathrm{dv}=\frac{h c^{3}}{8 \pi k v_{\mathrm{ul}}^{2}} A_{\mathrm{ul}} N_{u}\left(\mathrm{Kkm} \mathrm{s}^{-1}\right)$, where $T_{\mathrm{ul}}$ is the antenna temperature of the upper $(u)$ to lower (l) transition, $v_{\mathrm{ul}}$ is the transition frequency, $A_{\mathrm{ul}}$ the Einstein $A$-coefficient, and $N_{u}$ the column density of the upper level. In a uniform medium we can employ Eq. (1) with $N_{u}=f_{u} n(X) L$, where $n(X)$ is the density of the emitting species, $X, L$ the path length of the emission region, and $f_{u}$ is the fractional population of the upper level, to yield,

$I_{\mathrm{ul}}=\int T_{\mathrm{ul}} \mathrm{dv}=\frac{h c^{3}}{8 \pi k v_{\mathrm{ul}}^{2}} A_{\mathrm{ul}} f_{u} n(X) L\left(\mathrm{~K} \mathrm{~km} \mathrm{~s}^{-1}\right)$.

The ratio of the [O I] $63 \mu \mathrm{m}$ to [C II] $158 \mu \mathrm{m}$ intensity from the neutral gas of the PDR associated with $\mathrm{H}_{2}$ (we neglect the contribution from the low density $\mathrm{H}$ layer) is given by

$\frac{I([\mathrm{O} \mathrm{I}])}{I([\mathrm{C} \mathrm{II}])}=\frac{A_{12} \lambda(63)^{2}}{A_{3 / 2,1 / 2} \lambda(158)^{2}} \frac{f_{1}}{f_{3 / 2}} \frac{x(\mathrm{O})}{x\left(\mathrm{C}^{+}\right)}$,

assuming uniform conditions along the emitting region, and where $x(\mathrm{X})=n(\mathrm{X}) / n\left(\mathrm{H}_{2}\right)$ is the fractional abundance of species $\mathrm{X}$. Substituting for $A_{\mathrm{ij}}$ and $\lambda_{\mathrm{ij}}$ using the values in Schöier et al. (2005) in Eq. (3), we obtain

$\frac{I([\mathrm{OI}])}{I([\mathrm{C} \mathrm{II}])}=5.96 \frac{f_{1}}{f_{3 / 2}} \frac{x(\mathrm{O})}{x\left(\mathrm{C}^{+}\right)}$.

Equation (4) can be solved exactly as a function of $n\left(\mathrm{H}_{2}\right)$, $T_{k}$, and the fractional abundance ratio of $\mathrm{O}$ to $\mathrm{C}^{+}$. The carbon to oxygen ratio has been derived from UV absorption measurements and results vary somewhat from 1.5 to 2.1 (Sofia et al. 2004; Asplund et al. 2009). For convenience we adopt a value $x(\mathrm{O}) / x\left(\mathrm{C}^{+}\right)=2$. We further assume that the $[\mathrm{O} \mathrm{I}]$ emission from $\mathrm{H}$ I regions can be neglected compared to the $\mathrm{H}_{2}$ region, because the $\mathrm{H}$ I density is too low to contribute significantly to the overall emission. A useful form in which to display the solutions to Eq. (4) is to calculate $I([\mathrm{O} I]) / I([\mathrm{C} I \mathrm{II})$ for a set of constant 


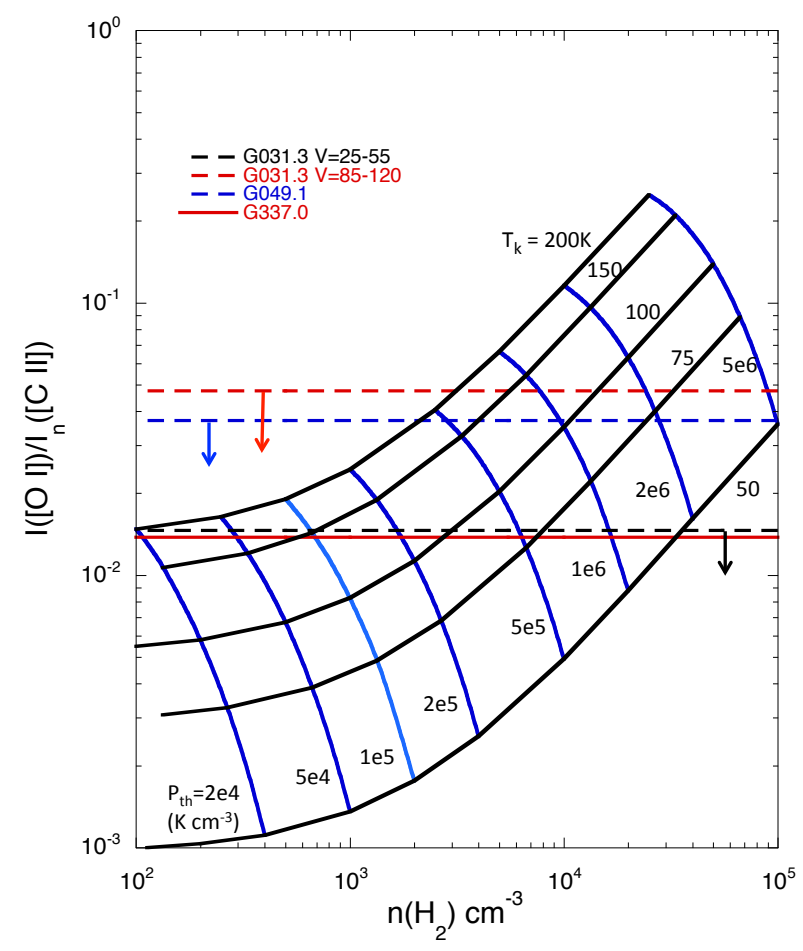

Fig. 2. Ratio of the $[\mathrm{OI}]$ intensity, $I([\mathrm{O} \mathrm{I}])$, to the $[\mathrm{C} \mathrm{II}]$ intensity arising from the neutral PDR region, $I_{n}([\mathrm{C} \mathrm{II}])$, as a function of $n\left(\mathrm{H}_{2}\right)$ for a set of constant thermal pressure, $P_{\text {th }}$, appropriate to [C II] emitting regions from PDRs (not from $\mathrm{H}^{+}$regions). The ratios for $P_{\mathrm{th}}=$ constant (solid blue lines) are labeled in units of $\mathrm{K} \mathrm{cm}^{-3}$. We limit the range of kinetic temperatures, $T_{k}$, appropriate to the [C II] emitting regions of PDRs to $50-200 \mathrm{~K}$. The solid black lines show the solutions for the ratio along $T_{k}=50,75,100,150$, and $200 \mathrm{~K}$. The horizontal lines represent the measured ratio (solid line) or measured limits (dashed lines) for the four LOS and velocity ranges discussed in the text. The LOS labels are indicated in the figure.

thermal pressures, $P_{\mathrm{th}}$, over a range of kinetic temperatures $T_{k}$ appropriate for PDRs.

Figure 2 shows the intensity ratio as a function of $n\left(\mathrm{H}_{2}\right)$ for different values of thermal pressure, $P_{\text {th }}$ (blue solid lines), ranging from $2 \times 10^{4}$ to $5 \times 10^{6} \mathrm{~K} \mathrm{~cm}^{-3}$, for kinetic temperatures in the range 50 to $200 \mathrm{~K}$, representing a lower bound on $T_{k}$ required for significant [C II] emission and an upper limit for standard PDRs. The solutions along constant $T_{k}$ are indicated by the set of solid black lines for $T_{k}=50,75,100,150$, and $200 \mathrm{~K}$. The model results in Fig. 2 use the collisional excitation rate coefficients of Jaquet et al. (1992) and assume an $\mathrm{H}_{2}$ ortho-to-para ratio of 3 (this ratio is not critical as the $\mathrm{O}$ de-excitation rates for collisions with ortho- and para- $\mathrm{H}_{2}$ with $\mathrm{O}$ are similar). For each value of $P_{\text {th }}$ (blue solid lines) the ratio drops as the temperature decreases from 200 to $50 \mathrm{~K}$.

In order to compare the model to the observed ratio of [O I] to [C II] we need to determine how much of the LOS [C II $]$ arises from the neutral gas, $I_{n}([\mathrm{C} \mathrm{II}])$. We adopt the procedure outlined in Langer et al. (2016) where the [N II] emission is used to calculated the contribution of the highly ionized gas to the total $[\mathrm{C} \mathrm{II}]$ intensity, $I_{\mathrm{tot}}([\mathrm{C} \mathrm{II}])$ and then solve for $I_{n}([\mathrm{C} \mathrm{II}])=I_{\text {tot }}([\mathrm{C} \mathrm{II}])-I_{\text {ion }}([\mathrm{CII}])$. As shown in Langer et al. (2016) $I_{\text {ion }}([\mathrm{C} \mathrm{II}]) \simeq 2.7 I([\mathrm{~N} \mathrm{II}])$ over a wide range of $n(\mathrm{e})$ appropriate to the LOS studied here. We plot $I([\mathrm{O} \mathrm{I}]) / I_{n}([\mathrm{C} \mathrm{II}])$ in Fig. 2 for the observed ratio (solid line) or 3- $\sigma$ limits (dashed lines) for four [C II] components. These measurements constrain the conditions in the PDRs, which are discussed below.

To solve for $P_{\text {th }}$ we only consider solutions with kinetic temperatures in the range $75-150 \mathrm{~K}$. The lower limit is consistent with the $[\mathrm{C} \mathrm{II}]$ intensity in these sources and the upper limit corresponds to the maximum predicted in PDRs without $\mathrm{HII}$ regions. For G337.0+0.0, where we have a 3.5- $\sigma$ detection, there are reasonable solutions only for $P_{\text {th }}$ in the range $5 \times 10^{4} \mathrm{~K} \mathrm{~cm}^{-3}$ $\left(T_{k} \sim 150 \mathrm{~K}, n\left(\mathrm{H}_{2}\right) \sim 330 \mathrm{~cm}^{-3}\right)$ to $\sim 5 \times 10^{5} \mathrm{~K} \mathrm{~cm}^{-3}\left(T_{k} \sim 75 \mathrm{~K}\right.$, $\left.n\left(\mathrm{H}_{2}\right) \sim 6.7 \times 10^{3} \mathrm{~cm}^{-3}\right)$. For the 3- $\sigma$ upper bound on $\mathrm{G} 031.3+0.0$ $V=25 \rightarrow 55 \mathrm{~km} \mathrm{~s}^{-1}$ we can only set an upper limit $P_{\text {th }} \lesssim$ $5 \times 10^{5} \mathrm{~K} \mathrm{~cm}^{-3}$. For the remaining two sources, G031.3+0.0 V = $85 \rightarrow 120 \mathrm{~km} \mathrm{~s}^{-1}$ and G049.1+0.0 the $3-\sigma$ limits on the ratio imply that $P_{\text {th }} \lesssim 5 \times 10^{6}$ and $2 \times 10^{6} \mathrm{~K} \mathrm{~cm}^{-3}$, respectively.

We can further constrain the conditions in the PDRs by deriving the $\mathrm{C}^{+}$column density, $N\left(\mathrm{C}^{+}\right)$, from $I([\mathrm{C} \mathrm{II}])$ as a function of $P_{\text {th }}$. Langer et al. (2014) showed that to a good approximation $N\left(\mathrm{C}^{+}\right)$is a simple function of $I([\mathrm{C} \mathrm{II}]) / P_{\text {th }}$ (Langer et al. 2014, see Eq. (4)) for low pressure PDRs. This relationship was later generalized to all pressure regimes (assuming optically thin emission) (Velusamy et al. 2017; Appendix B) and it is this form we use to calculate $N\left(\mathrm{C}^{+}\right)$as a function of $I([\mathrm{C} \mathrm{II}])$ and $P_{\mathrm{th}}$. We rewrite Eq. (19) from Velusamy et al. (2017) to solve for $N\left(\mathrm{C}^{+}\right)$ as a function of $P_{\mathrm{th}}$,

$N_{17}\left(\mathrm{C}^{+}\right)=\frac{1.96 \times 10^{4} I([\mathrm{C} \mathrm{II}])}{P_{\mathrm{th}}}+8.32 \times 10^{-2} I([\mathrm{C} \mathrm{II}])\left(\mathrm{cm}^{-2}\right)$,

where the column density, $N_{17}$, is expressed in units of $10^{17} \mathrm{~cm}^{-2}$. In Fig. 3, we plot $N\left(\mathrm{C}^{+}\right)$as a function of $P_{\text {th }}$ for the $[\mathrm{CII}]$ intensity from the neutral PDR, $I_{n}([\mathrm{C} \mathrm{II}])$, for the four sources listed in Table 2.

Models of PDRs by Wolfire et al. (2010) show that the column density of the $\mathrm{C}^{+}$layer, $N\left(\mathrm{C}^{+}\right)$is about $2-3 \times 10^{17} \mathrm{~cm}^{-2}$ roughly independent of mass, ambient radiation field, metallicity, and thermal pressure for a wide range of clouds typical of the ISM. Wolfire et al. (2010) consider clouds with $P_{\text {th }} \sim 10^{4}$ to $10^{5} \mathrm{~K} \mathrm{~cm}^{-3}$, based on the pressures derived from ${ }^{12} \mathrm{CO}$ and ${ }^{13} \mathrm{CO}$ (Sanders et al. 1993). If we assume $N\left(\mathrm{C}^{+}\right) \sim 2$ $3 \times 10^{17} \mathrm{~cm}^{-2}$ from the results of Wolfire et al. (2010) then the curves in Fig. 3 imply that $P_{\text {th }}$ for G337.0+0.0 and G031.3+ $0.0 V=25 \rightarrow 55 \mathrm{~km} \mathrm{~s}^{-1}$ lie in the range $2-5 \times 10^{5} \mathrm{~K} \mathrm{~cm}^{-3}$. While for G049.1+0.0 $P_{\mathrm{th}}=8 \times 10^{4}-1.2 \times 10^{5} \mathrm{~K} \mathrm{~cm}^{-3}$ and for $\mathrm{G} 031.3+0.0 \mathrm{~V}=85 \rightarrow 120 \mathrm{~km} \mathrm{~s}^{-1}$ we have $P_{\text {th }}=5 \times 10^{4}-$ $8 \times 10^{4} \mathrm{~K} \mathrm{~cm}^{-3}$.

Combining these limits with those derived from the [O I] to [C II] ratio provides additional constraints on $\mathrm{G} 337.0+0.0$, where we have detected [O I], and on G031.3+0.0 V=25 $\rightarrow 55 \mathrm{~km} \mathrm{~s}^{-1}$ where we have a good upper limit. For G337.0+0.0 the $P_{\text {th }}$ lies in the range $2 \times 10^{5} \mathrm{~K} \mathrm{~cm}^{-3}\left(T_{k} \sim 100 \mathrm{~K}, n\left(\mathrm{H}_{2}\right) \sim 2 \times 10^{3} \mathrm{~cm}^{-3}\right)$ to $5 \times 10^{5} \mathrm{~K} \mathrm{~cm}^{-3}\left(T_{k} \sim 75 \mathrm{~K}, n\left(\mathrm{H}_{2}\right) \sim 6.7 \times 10^{3} \mathrm{~cm}^{-3}\right)$. For $\mathrm{G} 031.3+0.0 \mathrm{~V}=25 \rightarrow 55 \mathrm{~km} \mathrm{~s}^{-1}$ the range of pressures is the same as for G337.0+0.0, but as we only have an upper limit on the $[\mathrm{O}$ I] to $[\mathrm{C} \mathrm{II}]$ ratio we can only set the following limits $T_{k} \leq 120 \mathrm{~K}$ and $n\left(\mathrm{H}_{2}\right) \leq 10^{4} \mathrm{~cm}^{-3}$. For the other two sources, where the limits on the [OI] to [C II] ratio are higher than for $\mathrm{G} 031.3+0.0 \mathrm{~V}=25 \rightarrow 55 \mathrm{~km} \mathrm{~s}^{-1}$ we can only constrain the thermal pressure using [O I]/[C II], as discussed above.

We can calculate the column density of oxygen, $N(\mathrm{O})$, from $I([\mathrm{O}])$ and the radiative transfer equation for the three fine structure levels (see the similar case for nitrogen discussed in Goldsmith et al. 2015) as a function of density and temperature. 


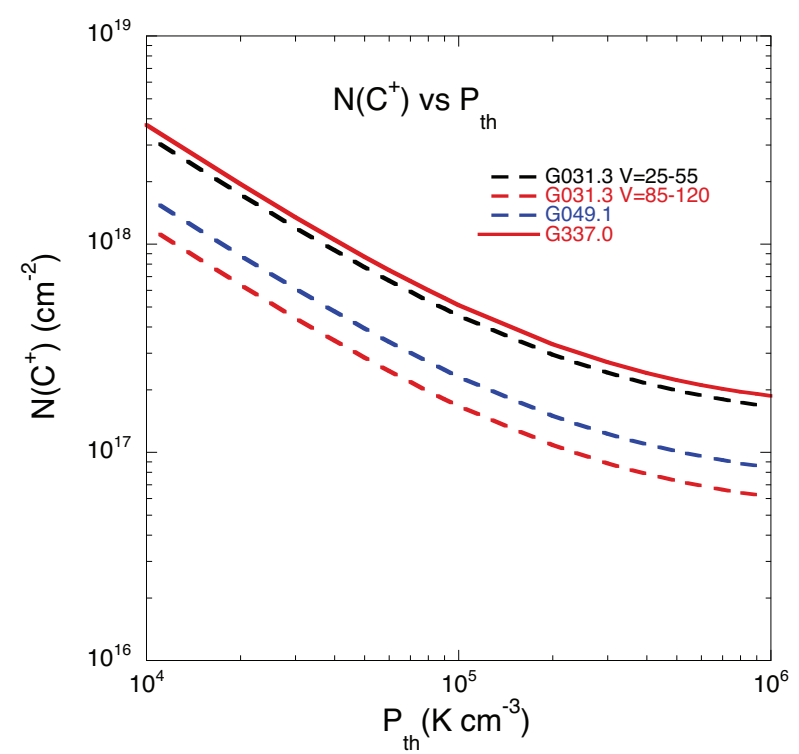

Fig. 3. Column density $N\left(\mathrm{C}^{+}\right)$of the [C II] emitting PDR layer is calculated from $I([\mathrm{C} I \mathrm{II}])$. The LOS are indicated by the insert labels.

For G337.0+0.0, assuming that the emission arises solely from the molecular gas, $N(\mathrm{O}) \sim 8 \times 10^{17}$ to $3 \times 10^{17} \mathrm{~cm}^{-2}$ over the pressure range 2 to $5 \times 10^{5}\left(\mathrm{~K} \mathrm{~cm}^{-3}\right)$ at $T_{k}=75 \mathrm{~K}$. For a typical inner galaxy fractional abundance of $\mathrm{O} / \mathrm{H}_{2}$ of $9 \times 10^{-4}$ the corresponding $\mathrm{H}_{2}$ column densities are $8.9 \times 10^{20}$ to $3.3 \times 10^{20} \mathrm{~cm}^{-2}$ and visual extinctions are $\mathrm{A}_{V} \sim 0.9$ to $0.4 \mathrm{mag}$. These values are well within the range of PDR models. Some of the [O I] emission can arise from the atomic hydrogen layer. Models of the $\mathrm{H}-\mathrm{to}-\mathrm{H}_{2}$ transition (Sternberg et al. 2014) predict that the gas becomes molecular within a column density $\sim(1-\mathrm{few}) \times 10^{20} \mathrm{~cm}^{-2}$. The collisional de-excitation rate coefficients for $\mathrm{H}$ on $\mathrm{O}$ are about 2.5 greater than those of $\mathrm{H}_{2}$ on $\mathrm{O}$ (Schöier et al. 2005) so the atomic layer could contribute significantly if the densities are high enough. If all the [O I] emission arises from the atomic gas, assuming the same thermal pressure in the $\mathrm{H}$ and $\mathrm{H}_{2}$ regions, we find $N(\mathrm{O}) \sim 3.2 \times 10^{17}$ to $1.2 \times 10^{17} \mathrm{~cm}^{-2}$ over the pressure range 2 to $5 \times 10^{5}\left(\mathrm{~K} \mathrm{~cm}^{-3}\right)$ at $T_{k}=75 \mathrm{~K}$. The corresponding atomic hydrogen column densities $N(\mathrm{H}) \sim 7 \times 10^{20}$ to $2.6 \times 10^{20} \mathrm{~cm}^{-2}$. With the limited information we have about the nature of the emission region, we cannot distinguish how much comes from each component, but the overall column density of $N(\mathrm{O})$ emitting gas most likely lies somewhere in between.

So far we have only used the intensities of [C II] and [O I], along with radiative transfer models, to constrain the density and pressure. However, the ratio $I([\mathrm{O} \mathrm{I}]) / I([\mathrm{C} \mathrm{II}])$ can also be used, along with PDR models, to constrain the intensity of the local UV field. Kaufman et al. (1999) have calculated this intensity ratio as a function of the cloud density, $n$, and the ratio of the incident far-ultraviolet (FUV) flux, $G_{o}$, at the PDR surface (where $G_{o}$ is in units of the Habing field, $1.6 \times 10^{-3} \mathrm{ergs} \mathrm{cm}^{-2} \mathrm{~s}^{-1}$ ) and the results are plotted in their Fig. 4. To compare our observed ratios (and limits) of $I([\mathrm{O} I]) / I([\mathrm{C} I \mathrm{I}])$ to the PDR models of Kaufman et al. (1999) we need to use the ratios in Table 3, which are expressed in similar units, $\mathrm{W} \mathrm{m}^{-2} \mathrm{sr}^{-1}$. For this comparison we use the ratio of $[\mathrm{O} \mathrm{I}]$ to $[\mathrm{C} \mathrm{II}]$ from the neutral gas, $I([\mathrm{O} \mathrm{I}]) / I_{n}([\mathrm{C} \mathrm{II}])$ (column 7 in Table 3).

In Table 3 column 8, we list the limits on the FUV field, $G_{o}$, derived from the observed $[\mathrm{O} \mathrm{I}]$ to $[\mathrm{C} \mathrm{II}]$ intensity and the PDR models of Kaufman et al. (1999) for the low end of the density range characteristic of our solutions, $2 \times 10^{3} \mathrm{~cm}^{-3}$ because it gives the maximum limit on $G_{o}$. The solutions for the [O I] detection in G337.0+0.0 and the limit on G013.3+0.0 $\left(V_{\mathrm{LSR}}=\right.$ $25 \rightarrow 55$ ) to constrain the FUV field intensity $G_{o}$, to about 15 or less, thus indicating that these sources are bathed in a low to moderate FUV field. The constraints on the other two sources restrict $G_{o}$ to be less than 50-100. By comparison, H II regions have much larger thermal pressures and values of $G_{o}$. For example, in $\mathrm{H}$ II regions, the $\mathrm{CO}$ spectral line energy distribution (CO SLEDs) (along with other tracers) along with PDR models (cf., Bron et al. 2018) have been used to derive $P_{\text {th }}$ and $G_{o}$ (Stock et al. 2015; Joblin et al. 2018; Wu et al. 2018). Among several sources studied, $P_{\text {th }}$ and $G_{o}$ range from $10^{6}$ to a few $\times 10^{8}\left(\mathrm{~K} \mathrm{~cm}^{-3}\right)$ and $10^{2}$ to $10^{8}$, respectively (Stock et al. 2015; Joblin et al. 2018; Wu et al. 2018), much larger than the values derived for our sources.

Based on our analysis of the thermal pressures and FUV field, the weak [OI] emission in G337.0+0.0 and the limits on [OI] emission in the other 3 sources, indicates that these sources are likely standard molecular clouds with PDRs and IBLs immersed in low to moderate UV fields $\left(G_{o} \leq 100\right)$, similar to those modeled by Wolfire et al. (2010), rather than strong $\mathrm{H}$ II regions in the references given above.

The presence of dense highly ionized gas, as derived from $[\mathrm{N}$ II], is an indication that the edge of the neutral gas does not transition rapidly to the low density WIM. Langer et al. (2017) have argued, based on [N II] and [C II] observations of the Scutum arm tangency, that the dense ionized gas is a result of the compression of the WIM as it falls into the gravitational well of the spiral arm. The widespread distribution of dense highly ionized gas in the Goldsmith et al. (2015) [N II] survey indicates that this component of spiral arms and evolved clouds is a general, but poorly understood, feature. More extensive surveys and maps of [C II] and [N II] are needed, along with observations of $[\mathrm{O}$ I], to understand these PDRs and their associated IBLs.

\section{Summary}

We observed the [OI] $63 \mu \mathrm{m}$ line at high spectral resolution using the GREAT $H$-band array on SOFIA towards four lines of sight with strong [N $\mathrm{II}]$ and $[\mathrm{C} \mathrm{II}]$ emission. Our goal was to use the $[\mathrm{O} \mathrm{I}]$ along with $[\mathrm{C} \mathrm{II}]$ to constrain the conditions in the PDRs associated with the highly ionized dense gas traced with [N II] emission arising from the adjacent IBLs. We detected [O I] at the 3.5- $\sigma$ level towards G337.0+0.0, but could only determine upper limits on the other sources. Comparing the ratio of [O I] to $[\mathrm{C} \mathrm{II}]$ and $N\left(\mathrm{C}^{+}\right)$to models incorporating the thermal pressure allowed us to place constraints on the thermal pressure, density, and kinetic temperature of the PDRs, even in some of the cases where we only had upper limits on [O I]. In two sources we were able to place tight constraints on the strength of the FUV radiation field, using the $[\mathrm{O} \mathrm{I}]$ to $[\mathrm{C} \mathrm{II}]$ ratio in conjunction with PDR models, $G_{o} \lesssim 15$. In these two sources the PDRs appear to be bathed in low to moderate FUV radiation fields. In the other two, the limits on the FUV field are larger, $G_{o}<50$ to 100 . Using these constraints on thermal pressure we derived a range of carbon and oxygen column densities, $N\left(\mathrm{C}^{+}\right)$and $N(\mathrm{O})$, consistent with PDR models. The weak to absent [O I] emission indicates that the PDRs are dense regions associated with standard molecular clouds immersed in moderate UV fields, and not H II regions.

Acknowledgements. We thank an anonymous referee for several very useful comments that improved our paper. We are grateful to the SOFIA engineering 
and operations teams for their support which enabled the observations presented here. The research reported here is based largely on observations made with the NASA/DLR Stratospheric Observatory for Infrared Astronomy (SOFIA) SOFIA is jointly operated by the Universities Space Research Association, Inc. (USRA), under NASA contract NAS2-97001, and the Deutsches SOFIA Institut (DSI) under DLR contract 50 OK 0901. This work was performed at the Jet Propulsion Laboratory, California Institute of Technology, under contract with the National Aeronautics and Space Administration. USA Government sponsorship acknowledged.

\section{References}

Abel, N. P., Ferland, G. J., Shaw, G., \& van Hoof P. A. M. 2005, ApJS, 161, 65 Asplund, M., Grevesse, N., Sauval, A. J., \& Scott, P. 2009, ARA\&A, 47, 481 Boreiko, R. T., \& Betz, A. L. 1996, ApJ, 464, L83

Bron, E., Agúndez, M., Goicoechea, J. R., \& Cernicharo, J. 2018, A\&A, submitted, [arXiv: 1801.01547]

Büchel, D., Pütz, P., Jacobs, K., et al. 2015, IEEE Trans. Terahertz Sci. Technol., 5,207

Eilers, P. H. C., \& Boelens, H. F. M. 2005, Leiden: Leiden University Medical Centre Report 2005, www . researchgate.net/publication/228961729

Goldsmith, P. F., Langer, W. D., Pineda, J. L., \& Velusamy, T. 2012, ApJS, 203, 13

Goldsmith, P. F., Yıldız, U. A., Langer, W. D., \& Pineda, J. L. 2015, ApJ, 814, 133

Guan, X., Stutzki, J., Graf, U. U., et al. 2012, A\&A, 542, L4

Heyminck, S., Graf, U. U., Güsten, R., et al. 2012, A\&A, 542, L1

Hollenbach, D. J., \& Tielens, A. G. G. M. 1997, ARA\&A, 35, 179

Hollenbach, D. J., \& Tielens, A. G. G. M. 1999, Rev. Mod. Phys., 71, 173

Jaquet, R., Staemmler, V., Smith, M. D., \& Flower, D. R. 1992, J. Phys. B At. Mol. Phys., 25, 285

Joblin, C., Bron, E., Pinto, C., et al. 2018, A\&A, 615, A129

Kaufman, M. J., Wolfire, M. G., Hollenbach, D. J., \& Luhman, M. L. 1999, ApJ, 527,795

Köhler, M., Habart, E., Arab, H., et al. 2014, A\&A, 569, A109

Langer, W. D., Velusamy, T., Pineda, J. L., et al. 2010, A\&A, 521, L17
Langer, W. D., Velusamy, T., Pineda, J. L., Willacy, K., \& Goldsmith, P. F. 2014, A\&A, 561, A122

Langer, W. D., Goldsmith, P. F., Pineda, J. L., et al. 2015, A\&A, 576, A1

Langer, W. D., Goldsmith, P. F., \& Pineda, J. L. 2016, A\&A, 590, A43

Langer, W. D., Velusamy, T., Goldsmith, P. F., et al. 2017, A\&A, 607, A59

Le Petit, F., Nehmé, C., Le Bourlot, J., \& Roueff, E. 2006, ApJS, 164, 506

Leurini, S., Wyrowski, F., Wiesemeyer, H., et al. 2015, A\&A, 584, A70

Mookerjea, B., Sandell, G., Vacca, W., Chambers, E., \& Guesten, R. 2018, A\&A, 616, A31

Oberst, T. E., Parshley, S. C., Nikola, T., et al. 2011, ApJ, 739, 100

Ossenkopf, V., Röllig, M., Neufeld, D. A., et al. 2013, A\&A, 550, A57

Pabst, C. H. M., Goicoechea, J. R., Teyssier, D., et al. 2017, A\&A, 606, A29

Pérez-Beaupuits, J. P., Stutzki, J., Ossenkopf, V., et al. 2015, A\&A, 575, A9

Pineda, J. L., Langer, W. D., Velusamy, T., \& Goldsmith, P. F. 2013, A\&A, 554, A103

Richter, H., Wienold, M., Schrottke, L., et al. 2015, IEEE Trans. Terahertz Sci. Technol., 5, 539

Risacher, C., Güsten, R., Stutzki, J., et al. 2016, A\&A, 595, A34

Sanders, D. B., Scoville, N. Z., Tilanus, R. P. J., Wang, Z., \& Zhou, S. 1993, in Back to the Galaxy, eds. S. S. Holt, \& F. Verter AIP Conf. Ser., 278, 311

Schneider, N., Güsten, R., Tremblin, P., et al. 2012, A\&A, 542, L18

Schöier, F. L., van der Tak, F. F. S., van Dishoeck, E. F., \& Black, J. H. 2005, A\&A, 432, 369

Sofia, U. J., Lauroesch, J. T., Meyer, D. M., \& Cartledge, S. I. B. 2004, ApJ, 605, 272

Sternberg, A., \& Dalgarno, A. 1989, ApJ, 338, 197

Sternberg, A., Le Petit, F., Roueff, E., \& Le Bourlot J. 2014, ApJ, 790, 10

Stock, D. J., Wolfire, M. G., Peeters, E., et al. 2015, A\&A, 579, A67

Tielens, A. G. G. M., \& Hollenbach, D. 1985, ApJ, 291, 722

Velusamy, T., Langer, W. D., Goldsmith, P. F., \& Pineda, J. L. 2017, ApJ, 838, 165

Wiesemeyer, H., Güsten, R., Heyminck, S., et al. 2016, A\&A, 585, A76

Wiesenfeld, L., \& Goldsmith, P. F. 2014, ApJ, 780, 183

Wolfire, M. G., Hollenbach, D., \& McKee, C. F. 2010, ApJ, 716, 1191

Wu, R., Bron, E., Onaka, T., et al. 2018, A\&A, in press, DOI: 10. 1051/0004-6361/201832595

Young, E. T., Becklin, E. E., Marcum, P. M., et al. 2012, ApJ, 749, L17 